\title{
Telegeriatrics: a possibility and a challenge
}

\section{Wahaba HMF}

\author{
Geriatric Medicine \& Gerontology department, Faculty of Medicine, Ain Shams University, Cairo, Egypt.
}

Population ageing is a triumph of development. We celebrate rising life expectancy as one of humanities' major achievement. Yet opportunity comes with challenge. Population ageing presents social, economic and cultural challenges to individuals, families, societies and global community. Globally, the number of elderly increased from $9.2 \%$ in 1990 to $11.7 \%$ in 2013 and will continue to grow as a proportion of the world population reaching $21.1 \%$ by 2050 .

Unfortunately, the transition to an ageing population is more rapid in developing countries, which also have fewer resources than the developed countries to meet the challenges of an ageing population.

The absolute number of people aged 65 and above in the Arab world has already doubled from 5.7 million in 1980 to 10.4 million in 2000, and is expected to increase to 21.3 million by 2020 . According to the percentages documented in 2013 Lebanon has the highest percentage of elderly; $10 \%$ followed by Egypt; $6.9 \%$.

The challenge is great; there is a great mismatch between resources and needs at all levels of care to elderly, their needs outweigh the available resources. Telemedicine/telehealth has been implemented worldwide for some time.

Telemedicine is emerging as a critical component of the healthcare crisis solution. Telemedicine holds the promise to significantly impact some of the most challenging problems of our current healthcare system: access to care, cost effective delivery, and distribution of limited providers (as the case of geriatrics consultants). Telemedicine can change the current paradigm of care and allow for improved access and improved health outcomes in cost effective ways.

Telegeriatrics is the extension of telemedicine to the elderly. The elderly's combination of limited mobility and chronic health problems often makes this group ideal beneficiary of distance medicine.

Telegeriatrics increases access to healthcare by providing remote patients with healthcare services and so can considerably decrease mortality, complications, decrease number of patients in waiting rooms, decrease ER visits, decrease hospital admissions, days of hospital stay and hence healthcare costs.

It can allow elderly to get access to specialized geriatrics care in the form of comprehensive multidisciplinary care, more frequent follow up through remote home care and so improve morbidities and quality of life. National studies in the USA showed that home monitoring of chronic diseases has reduced hospital visits by as much as $50 \%$ by keeping patients stable through daily monitoring. The average for readmission to hospitals within 30 days following a heart failure episode was $20 \%$.

Telehealth monitoring programs have reduced that level to less than $4 \%$. Timely provision of treatments that effectively reverse the consequences of a stroke have risen from $15 \%$ to $85 \%$ due to the availability of telestroke programs.

It also provides better documentation and a centre for data for the geriatrics population that can guide policy makers and healthcare providers. Patients can stay in their local communities and, when hospitalized away from home, can keep in contact with family and friends. Moreover, it is more convenient and less costly for the patient.

Telemedicine can support educational programs with blended onsite and online courses that can target both healthcare professionals (CME, specialist training programs) and recipients of the care (elderly and their caregivers).

Telemedicine has indirect benefits as the reduction in extended travel to obtain necessary care which reduces the related carbon footprint. Moreover, employees can avoid absences through participating remotely in consults.

Unfortunately, telemedicine has lagged behind its potential for a number of reasons. ICT ignorance has been one of the most important factors fighting the implementation of such indispensable services. Another 
very influential factor is the difficulty of incorporating the service in the current healthcare system. Multiple other notable factors include inconsistent electricity and internet services in rural areas, trust and privacy issues, competition with the private sector and the relatively high startup cost.

Despite the evidence in literature showing the benefits of telemedicine in general and in telespecialities, healthcare providers and policymakers are reluctant to implement such a system. It has not been only notable in developing countries, where the challenges for such a change are great, but even in developed countries where such a concept has been introduced a couple of decades ago. It seems prudent to investigate ways to overcome the challenges rather that display benefits that have been established. 